\title{
CBASP@home: Ein internetbasiertes Situationsanalysen- Training zur Stabilisierung des Therapieerfolgs nach stationärer Therapie für chronisch depressive Patienten
}

\author{
Eva-Lotta Brakemeier ${ }^{\mathrm{a}} \quad$ Janina Marchner $^{\mathrm{a}} \quad$ Simone Gutgsell ${ }^{\mathrm{b}} \quad$ Vera Engel $^{\mathrm{c}} \quad$ Martina Radtke $^{\mathrm{c}}$ \\ Brunna Tuschen-Caffier ${ }^{d}$ Claus Normann $^{c}$ Matthias Berking ${ }^{\mathrm{e}}$ \\ a Psychologische Hochschule Berlin, Deutschland \\ ${ }^{\mathrm{b}}$ Universitäre Psychiatrische Kliniken Basel, Schweiz \\ ${ }^{c}$ Abteilung für Psychiatrie und Psychotherapie, Universitätsklinikum Freiburg, \\ ${ }^{d}$ Abteilung für Klinische Psychologie und Psychotherapie, Universität Freiburg, \\ e Abteilung für Klinische Psychologie und Psychotherapie, Philipps-Universität Marburg, Deutschland
}

\section{Schlüsselwörter}

Internetbasierte Psychotherapie - Chronische Depression . CBASP $\cdot$ Situationsanalyse $\cdot$ Rückfallprophylaxe

\section{Zusammenfassung}

Hintergrund: Aufbauend auf einer ambulanten CBASP-Behandlung wurde ein stationäres multidisziplinäres Konzept zur Behandlung chronischer Depression entwickelt. Eine Pilotstudie zeigte positive Ergebnisse hinsichtlich der Wirkung dieses Konzepts, jedoch berichteten einige Patienten, dass die 12-wöchige Therapie zu kurz und die Zeit unmittelbar nach Entlassung schwierig sei. Daher wurde CBASP@home als ein internetbasiertes Situationsanalysen (SA)-Training zur Stabilisierung des erzielten Behandlungserfolgs konzipiert. CBASP@home ist eine 3-monatige Step-down-Onlineintervention. Die Patienten werden dabei unterstützt, die erlernten SA zu Hause fortzuführen und in den Alltag zu transferieren. An insgesamt 9 Terminen füllten die Patienten das SA-Formular über einen Sicherheitsserver aus und erhielten von ihrem stationären Psychotherapeuten zeitnah Rückmeldung. Fallbericht: In der vorliegenden Phase-I-Studie wurde die Akzeptanz und Durchführbarkeit von CBASP@home an 2 Einzelfällen untersucht. CBASP@home wurde von beiden Patienten sehr gut angenommen und als sinnvolle Unterstützung bewertet. Zudem konnte bei beiden Patienten eine zunehmende Leistungssteigerung in der SA-Durchführung bei gleichzeitig rückläufiger depressiver Symptomatik bis zur Remission beobachtet werden. Schlussfolgerungen: Die beiden Einzelfallauswertungen liefern Hinweise, dass CBASP@ home Transferprozesse vom stationären Setting in den Alltag erleichtern, Behandlungserfolge beibehalten bzw. Rückfällen vorbeugen kann. Wenn sich diese positiven Erfahrungen in zukünftigen Studien bestätigen sollten, kann CBASP@home wesentlich zur Verbesserung der langfristigen Versorgung chronisch depressiver Patienten beitragen.

\section{Keywords}

Internet-based psychotherapy - Chronic depression . CBASP - Situational analysis · Relapse prevention

\section{Summary}

CBASP@home: An Internet-Based Situational Analysis

Training as a Maintenance Treatment after Inpatient Therapy for Chronically Depressed Patients

Background: On the basis of an outpatient CBASP treatment an inpatient multidisciplinary concept was developed in order to treat chronic depression. A pilot study showed positive outcome measures of this concept. Nevertheless, some patients reported that the 12-week treatment was too short and the time right after discharge was difficult. Therefore, CBASP@home was designed as an internet-based situational analysis (SA) training in order to stabilize the reached therapeutic success. CBASP@home is a 3-month step down online intervention. The patients are supported in continuing the learned SA at home and in transferring them in their daily routine. At 9 visits the patients filled out the SA form through a security server and received feedback from their impatient therapist shortly after. Case Report: In the present phase I study the acceptance and feasibility of CBASP@home was examined in 2 cases. CBASP@ home has been very well accepted by both patients and evaluated as a very sensible support. Moreover, in both cases an improved SA performance could be observed and at the same time the depression symptoms decreased to remission. Conclusions: The analyses of the 2 individual case reports indicate that CBASP@home can facilitate transfer processes from an inpatient setting into daily routine, maintain treatment success, and prevent relapses. If these positive experiences will be approved in future studies, CBASP@home can considerably contribute to an improvement of a long-lasting supply of chronically depressed patients.

\section{KARGER \\ Fax +49 7614520714 \\ Information@Karger.com}

www.karger.com (c) 2013 S. Karger GmbH, Freiburg

1016-6262/13/0233-0190\$38.00/0

Accessible online at:

www.karger.com/ver
Prof. Dr. Eva-Lotta Brakemeier

Psychologische Hochschule Berlin

Am Köllnischen Park 2, 10179 Berlin, Deutschland

e-l.brakemeier@psychologische-hochschule.de 


\section{Einleitung}

Chronische Depression ist eine weitverbreitete psychische Störung, die die Lebensqualität stark beeinträchtigt. Schätzungsweise 1,2 Millionen Menschen leiden derzeit in Deutschland an dieser psychischen Störung [Arnow und Constantino, 2003; Brakemeier et al., 2012a; Dunner, 2001]. Chronische Depression zeichnet sich durch kognitive, affektive, sozial-motivationale und vegetative Symptome über einen mindestens 2 Jahre andauernden Zeitraum aus. Sie verringert die berufliche und soziale Funktionsfähigkeit und geht sowohl für die Betroffenen als auch für deren Angehörige mit einer großen Belastung einher [Keller et al., 2000; Ley et al., 2009; Schweiger et al., 2007]. Chronisch depressive Patienten gelten im klinischen Alltag im Vergleich zu episodisch depressiven Patienten als schlechter behandelbar und werden aufgrund niedriger Responseraten oft als «therapieresistent» bezeichnet [Bschor, 2008; Helbig-Lang und Petermann, 2009; Ley et al., 2009].

James McCullough [2000] postuliert, dass bei chronisch depressiven Patienten die häufig erlebten traumatisierenden $\mathrm{Be}$ ziehungserfahrungen (vor allem emotionaler Missbrauch und emotionale Vernachlässigung, vgl. z.B. Riso et al. [2002]) die Entwicklung der operationalen Denkweise [Piaget, 1981] blockieren. Gemäß McCullough äußert sich dieses Entwicklungsdefizit kognitiv in monologisierendem Sprechen, präkausaler Denkweise und geringer Beeinflussung des Denkens durch Rückmeldungen der Umwelt, was korrigierende Beziehungserfahrungen erschwert. Emotionale Defizite zeigen sich in Form von gering ausgeprägter Empathie, Egozentrik und Schwierigkeiten der Emotionskontrolle in Stresssituationen, weshalb chronisch Depressive starke Auffälligkeiten im interpersonellen Bereich aufweisen [Brakemeier et al., 2012a]. Um den vielschichtigen Problemen chronisch depressiver Patienten gerecht zu werden, entwickelte McCullough daher ein störungsspezifisches Behandlungskonzept, welches integrativ und Schulen übergreifend kognitive, verhaltenstherapeutische, interpersonelle und psychodynamische Strategien integriert. Folgende spezifische Therapiestrategien sind im Cognitive Behavioral Analysis System of Psychotherapy (CBASP) enthalten [Brakemeier und Normann, 2012]:

Kindheits- und Lebenserfahrungen werden durch die Liste prägender Bezugspersonen (LpB) in einen Zusammenhang zu den heutigen interpersonellen Problemen, darunter auch Problemen mit Therapeuten, gestellt. Daher werden Übertragungshypothesen (ÜH) bereits in der ersten Phase der Therapie pro-aktiv und für den Patienten transparent entwickelt. Als innovative Art der therapeutischen Beziehungsgestaltung lässt sich das disziplinierte persönliche Einlassen (DPE) bezeichnen, das durch das Preisgeben eigener Gefühle und Reaktionen eine auf die Bedürfnisse chronisch Depressiver adaptierte Rolle des Therapeuten und eine menschliche therapeutische Beziehung ermöglicht. In Verbindung damit stehen interpersonelle Diskriminationsübungen (IDÜ), durch die den Patienten transparent gemacht wird, dass die Therapeu- ten anders - häufig entgegengesetzt - als die missbrauchenden, vernachlässigenden, negativ prägenden Bezugspersonen aus ihrem bisherigen Leben reagieren, woraus heilsame Beziehungserfahrungen resultieren können. Als zentrales Element gilt schließlich die Situationsanalyse (SA) mit Verhaltenstrainings durch Rollenspiele, kognitive Umstrukturierung und Transferübungen. Zudem fließen interpersonelle Strategien durch die Nutzung des Kiesler-Kreises (unter anderem zur Gestaltung der therapeutischen Beziehung) ein. In Abbildung 1 sind die genannten CBASP-Strategien und ihre Verbindungen zusammenfassend dargestellt. Eine ausführliche Darstellung aller Strategien findet sich z.B. bei McCullough [2000] oder Brakemeier und Normann [2012]. Da SA die Hauptstrategie der Onlineintervention ist, beschränken sich die weiteren Ausführungen auf die ausführliche Darstellung dieser Strategie.

McCullough versteht CBASP in erster Linie als «Lerntherapie» (acquisition learning), wobei das Ziel der Therapie im Erlernen neuer Verhaltens- und Denkstrategien sowie dem Erwerb interpersoneller Fertigkeiten liegt. Darüber hinaus wird das transparente Erlernen bzw. Erleben neuer korrigierender Beziehungserfahrungen zur Heilung der traumatisierenden Beziehungserfahrungen aus der Kindheit angestrebt. Daher lässt sich CBASP am besten als «interpersonelle Lerntherapie» verstehen.

Seitdem die Wirksamkeit von CBASP durch eine große randomisierte kontrollierte multizentrische ambulante Studie [Keller et al., 2000] belegt wurde, weckt CBASP zunehmend weltweites Interesse. Da eine Subgruppe chronisch depressiver Patienten jedoch so schwer beeinträchtigt ist, dass sie von ambulanten Therapien nicht zufriedenstellend profitiert und stationär behandelt werden muss, wurde CBASP als modifiziertes stationäres interdisziplinäres Konzept auf der Schwerpunktstation für affektive Störungen des Universitätsklinikums Freiburg i.Br., Abteilung für Psychiatrie und Psychotherapie, implementiert und evaluiert [Brakemeier et al., 2011; Brakemeier und Normann, 2012]. Eine gerade abgeschlossene Phase-II-Studie mit 70 Patienten zeigte positive Ergebnisse hinsichtlich der Wirksamkeit von CBASP, da $82 \%$ auf die 12-wöchige Therapie ansprachen, wovon $42 \%$ die Remission erreichten. Allerdings respondierten somit $18 \%$ auch nicht auf diese intensive Therapie, wobei bei keinem der Patienten eine Verschlechterung des Gesundheitszustands festgestellt werden konnte (Brakemeier et al., 2013, persönliche Mitteilung). Einige Patienten - insbesondere die Non-Responder - berichteten bei Therapieende bzw. den Katamnese-Befragungen jedoch, dass die stationäre Therapie zu kurz und die Zeit unmittelbar nach Entlassung besonders schwierig sei. Erste Prädiktor-Analysen zeigten, dass die Non-Responder im Vergleich mit den Respondern mehr Persönlichkeitsstörungen aufwiesen, unzufriedener mit dem sozialen Netz waren sowie häufiger isoliert (d.h. ohne Lebenspartner) lebten (Brakemeier et al., 2013, persönliche Mitteilung). Die Notwendigkeit einer Behandlung der chronischen Depression über den statio- 


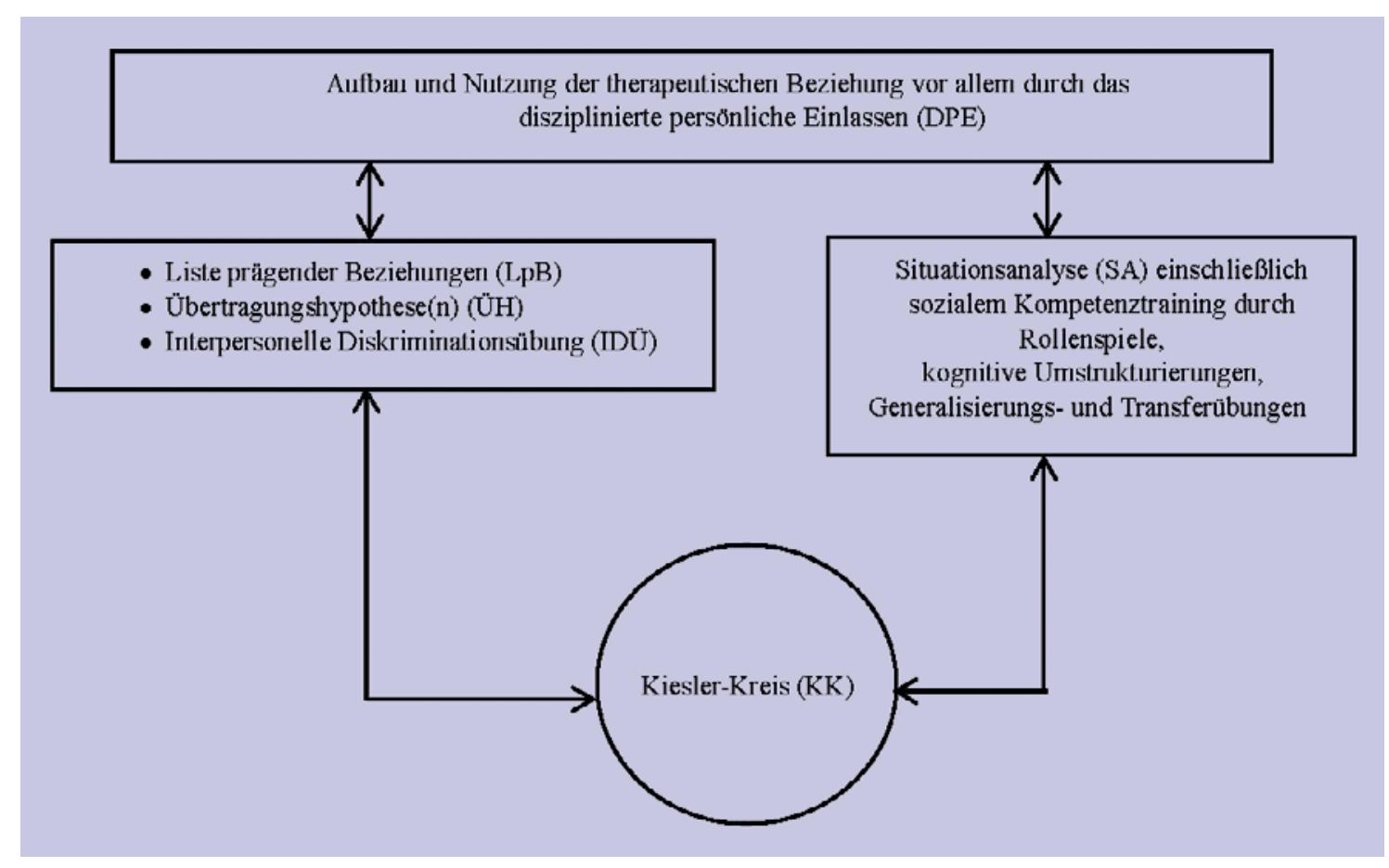

Abb. 1. Elemente der Psychotherapie der chronischen Depression [Brakemeier et al., 2012a].

nären Rahmen hinaus ergibt sich - abgesehen von den berichteten Erfahrungen - aus dem häufig rezidivierenden Verlauf der Depression und der Tatsache, dass teilremittierte Patienten deutlich häufiger einen Rückfall erleiden als vollremittierte Patienten [vgl. de Jong Meyer et al., 2007]. Da vermutet werden kann, dass CBASP bei einer längeren Therapiedauer besser wirkt [Brakemeier und Normann, 2012; Cuijpers et al., 2010], jedoch Krankenkassen häufig keine längeren stationären Aufenthalte finanzieren, erscheint gerade für die Non-Responder eine Verlängerung der Behandlungsdauer im ambulanten Setting vielversprechend. Eine Befragung zum Bedarf einer internetbasierten Nachsorge unter 33 ehemaligen stationär behandelten CBASP@5-Patienten (CBASP-Hauptbehandlung) zeigte, dass $85 \%$ auch Interesse an einer derartigen Intervention hätten (Gutgsell, 2012, persönliche Mitteilung). Zudem konnte eine Studie im ambulanten Setting zeigen, dass sich CBASP als längerfristige Erhaltungstherapie für chronisch depressive Patienten eignet, die zuvor auf eine ambulante CBASP-Therapie angesprochen haben [Klein et al., 2004].

Trotz Bemühungen, CBASP auch in Deutschland im ambulanten Setting flächendeckend zu disseminieren, gibt es bis dato insbesondere in ländlichen Gegenden relativ wenige in CBASP geschulte ambulant arbeitende Psychotherapeuten mit freien Therapieplätzen, weshalb sich eine Onlinevariante anbietet. Zudem sind die Patienten durch diese Intervention gefordert, die im «Schonraum» des stationären Rahmens neu gelernten Erlebens- und Verhaltensmuster nach Entlassung unter den Alltagsbelastungen als «Hausaufgabe» allein anzuwenden. Nach einer CBASP-Behandlung sollten insbesondere die situativen und interpersonellen Fertigkeiten konsequent weiterhin trainiert werden, um so ein erneutes Abrut- schen in den Teufelskreis der Hilflosigkeit und chronischen Depression zu verhindern [vgl. Brakemeier et al., 2012a]. Der Transferprozess in den Alltag erfordert seitens des Patienten eine hohe Motivation, Anpassungsleistung, Selbstaufmerksamkeit und Zielorientierung. Gleichzeitig birgt die Zeit nach der Entlassung viele Herausforderungen: Neben den Alltagsbelastungen können Erwartungshaltungen und Widerstände des häuslichen Umfeldes den Transferprozess erschweren. Mögliche «Nebenwirkungen» einer Psychotherapie wie Veränderungen im psychosozialen Bereich [Brakemeier et al., 2011, 2012b] gilt es zu Hause zu bewältigen. Das Ausbleiben erwünschter Zielzustände (z.B. Wohlbefinden) kann die Veränderungsmotivation zusätzlich reduzieren. Folglich werden die Patienten oft mit der Empfehlung und/oder auf eigenen Wunsch entlassen, die Therapie im ambulanten Rahmen fortzusetzen (Gutgsell, 2012, persönliche Mitteilung).

Die bereits erwähnte therapeutische Unterversorgung führt dazu, dass Patienten in der Zeit nach der stationären Therapie, die durch ein erhöhtes Rückfallrisiko und oftmals psychosoziale Unsicherheiten geprägt ist, auf sich allein gestellt sind. Um einerseits dem notwendigen therapeutischen Bedarf, andererseits auch der Knappheit therapeutischer und finanzieller Ressourcen gerecht zu werden, scheinen gestufte Präventions- und Behandlungsansätze (Stepped Care) von zunehmender Bedeutung. Gemäß des «Stepped Care»-Ansatzes [Haaga, 2000] soll jeder Patient genauso viel Unterstützung, Beratung und Therapie bekommen wie er idealerweise benötigt. Demzufolge sollen intensivere Angebote (und somit in der Regel auch teurere) auf weniger intensive Angebote folgen (Step-up) und weniger intensive Behandlungskomponenten sollen an intensivere Angebote (Step-down) anschließen [vgl. Bauer et al., 
2008; Cukrowicz und Joiner, 2007]. Der Einsatz traditioneller und neuer Informations- und Kommunikationstechnologien (z.B. Telefon, Computer, Internet-Chat, E-Mail, SMS) bietet die Möglichkeit, Patienten nach einer stationären Therapie im Sinne eines Step-down-Angebots zu erreichen und so den Transferprozess vom stationären Setting in den Alltag zu erleichtern, die während des stationären Aufenthalts erzielten Behandlungserfolge zu stabilisieren und Rückfälle zu verhindern. Das Ziel, die Lücken zwischen stationärer und ambulanter Versorgung zu überbrücken, wird in Deutschland derzeit insbesondere in der Heidelberger Arbeitsgruppe um Hans Kordy verfolgt [z.B. Bauer et al., 2008, 2011; Kordy et al., 2006; Kordy, 2013; Wolf et al., 2006; Zimmer et al., 2010].

Computerbasierende Interventionen bieten zudem - in Abhängigkeit von der Art der Darbietung - den Vorteil der zeitlichen Flexibilität, der Kostenersparnis, der einfachen Erreichbarkeit, der Symptomüberwachung und der Förderung der Eigenverantwortlichkeit und somit auch der Eigenständigkeit. Zusätzlich kann die während des stationären Rahmens aufgebaute therapeutische Beziehung weiterhin genutzt werden. Dies dürfte insbesondere in der Zeit unmittelbar nach Entlassung hilfreich sein, da so die therapeutischen Ziele nahtlos weiterverfolgt werden können. Die SA, der eine potenzielle Wirksamkeit zugesprochen wird, scheint mit ihrer Manualisierung durchaus zur Implementierung in eine einfach zu bedienende computerbasierende Darbietungsform geeignet zu sein. Da es bislang keine Onlineintervention gibt, die auf eine stationäre CBASP-Therapie aufbaut, könnte «CBASP@home» diese Versorgungslücke schließen (Gutgsell, 2012, persönliche Mitteilung).

\section{Beschreibung von CBASP@home}

CBASP@home ist konzipiert als Teil eines gestuften Behandlungsplans in der therapeutischen Versorgung chronisch depressiver Patienten. Die CBASP-spezifische Step-down Onlineintervention mit Psychotherapeutenkontakt schließt an die stationäre CBASP-Therapie an. Es wird angenommen, dass der Patient durch die intensive stationäre CBASP-Therapie (insbesondere die Anwendung der Strategien DPE und IDÜ, siehe oben) heilsame korrigierende Beziehungserfahrungen mit seinem Einzeltherapeuten, aber auch anderen Teammitgliedern und Mitpatienten sammelt, sodass er bei Entlassung seinen Mitmenschen wieder mit mehr Vertrauen, Offenheit und Selbstbewusstsein begegnen kann [vgl. Brakemeier und Normann, 2012]. Auf Basis dieser Beziehungserfahrungen und insbesondere der bestehenden therapeutischen Beziehung zum Einzeltherapeuten soll CBASP@home die Patienten nach Entlassung darin unterstützen, die während der stationären Therapie erlernte Strategie der SA zu Hause weiter fortzuführen und in den Alltag zu transferieren. Die SA nimmt üblicherweise ca. 75\% der Psychotherapiezeit in Anspruch. Diese Strategie wurde für CBASP@home gewählt, weil sie als eine der wichtigsten therapeutischen CBASP-Techniken gilt und gut in einer Onlineintervention umsetzbar ist. Daher wird die SA im Folgenden näher beschrieben und anhand von 2 anschaulichen Fallbeispielen dargestellt.

Die SA ist eine strukturierte, mehrstufige, interpersonelle, funktionale Situation-Reaktion-Konsequenz-Technik, die Verhaltenstraining durch Rollenspiele, kognitive Umstrukturierung und Transferübungen integriert. Die Wurzeln der SA liegen in der Verhaltensanalyse wie sie vor allem von Kanfer und Saslow [1976] vertreten wurde [vgl. Brakemeier und Hautzinger 2008; Brakemeier et al., 2012a; Schweiger und Sipos, 2009]. Im Rahmen des CBASP-Konzepts soll die SA chronisch depressive Patienten darin unterstützen, Verhaltenskonsequenzen in interpersonellen Beziehungen zu erkennen und die Gedanken sowie das Verhalten entsprechend persönlichen realistischen Zielen und Ressourcen auszurichten. Die Patienten sollen also lernen, dass ihr Verhalten - entgegen ihrer Erwartungen bzw. früheren Lernerfahrungen - Konsequenzen hat («wahrgenommene Funktionalität»), wodurch sie aus der erlernten chronischen Hilf- und Hoffnungslosigkeit herauskommen. Da die Aufmerksamkeit des Patienten auf eine konkrete Situation gelenkt wird, soll zudem das globale und transsituationale prä-operationale Denken allmählich aufgelöst werden, sodass der Patient beispielsweise logischer und situativ zu denken lernt, seine egozentrische Sichtweise minimiert und empathisch mit seiner Umwelt umgeht.

Bei der Durchführung der SA wird der Leidensdruck durch Verschärfung der Problematik in der Situation bewusst erhöht, um dann im Laufe der SA eine spürbare Abnahme des Leidensdrucks bis hin zum sogenannten Erleichterungsmoment durch angemessenes Problemlösen zu erzeugen (negative Verstärkung). In der 1. Phase der SA - der Explorationsphase (Tab. 1 und 2) - beschreibt der Patient im 1. Schritt (Situationsbeschreibung) ein problematisches, interpersonelles Ereignis aus der jüngsten Vergangenheit mit einem klar definierten Anfangs- und Endpunkt, also einem konkret umschriebenen Zeitabschnitt (slice of time). Nach dieser rein deskriptiven Beschreibung soll der Patient im 2. Schritt (Interpretationen) seine relevanten Interpretationen bzw. Bewertungen der Situation festhalten. Im 3. Schritt (Verhalten) erfolgt dann die Erfassung des vom Patient gezeigten Verhaltens, wobei sein Verhalten auch im Kiesler-Kreis eingeordnet werden kann. Anschließend definiert er im 4. Schritt sein tatsächlich erreichtes Ergebnis (TE, actual outcome), wobei er im 5. Schritt überlegt, ob er dieses oder ein anderes Ergebnis erreichen wollte. Im letzteren Fall formuliert er ein neues erwünschtes Ergebnis (EE, desired outcome) der Situation, das er realistisch umsetzen können und das in ihm verankert sein muss. Die häufig erlebte Diskrepanz zwischen dem tatsächlich erreichten und dem eigentlich erwünschten Ergebnis (6. Schritt) soll beim Patienten zunächst zu erlebbarem Unbehagen und somit zur Voraussetzung negativer Verstärkung führen, weil daraus die eigene Änderungsmotivation erwachsen kann. Dementsprechend bespricht der Psychotherapeut 
Tab. 1. Fallbeispiel Herr Schmidt: Eine SA aus der 2. Woche der CBASP@home-Intervention (PPRF-Leistung=2)

\begin{tabular}{|c|c|c|c|}
\hline Schritte der SA & Herr Schmidt & Kommentar der Psychotherapeutin & PPRF-Wert \\
\hline \multicolumn{4}{|l|}{ I. Explorationsphase } \\
\hline 1. Situationsbeschreibung & $\begin{array}{l}\text { Meine Frau und ich sitzen am Frühstücks- } \\
\text { tisch. Sie sagt: «Heute Abend möchte ich } \\
\text { zur Kantorei und es wäre doch schön, } \\
\text { wenn Du endlich auch mal wieder } \\
\text { mitkommst. Zudem sollten wir vorher } \\
\text { unseren Sohn und all die Enkelkinder } \\
\text { besuchen. Okay?» Ich schweige kurz, } \\
\text { schlucke und sage: «Na gut.» }\end{array}$ & $\begin{array}{l}\text { Lieber Herr Schmidt, diesen Schritt haben Sie } \\
\text { sehr gut bearbeitet: Sie haben eine wichtige } \\
\text { interpersonelle Situation ausgesucht und einen } \\
\text { klaren Anfangs- und Endpunkt bestimmt. } \\
\text { Zudem haben Sie die Situation aus der } \\
\text { Beobachterperspektive klar beschrieben: } \\
\text { Ganz toll! }\end{array}$ & 1 \\
\hline 2. Interpretation & $\begin{array}{l}\text { 1. Ich will heute nicht beides machen: } \\
\text { Besuch beim Sohn und Kantorei - } \\
\text { das wird mir zu viel. } \\
\text { 2. Ich kann mich nicht wehren. } \\
\text { 3. Ich darf nicht nein sagen, sonst denkt } \\
\text { sie wieder, ich sei depressiv. }\end{array}$ & $\begin{array}{l}\text { Die erste Interpretation ist sehr gut, da es ja } \\
\text { wirklich so war und diese auch hilfreich ist für ein } \\
\text { gutes EE. Die beiden anderen Interpretationen } \\
\text { sind für mich zwar nachvollziehbar, da ich Sie } \\
\text { und Ihre Prägungen ja gut kenne, doch werden } \\
\text { wir weiter unten feststellen, dass beide nicht in } \\
\text { der Situation verankert und nicht hilfreich für ein } \\
\text { gutes erwünschtes Ergebnis sind. }\end{array}$ & 0 \\
\hline 3. Verhalten & $\begin{array}{l}\text { Ich höre zu, schaue betreten auf meinen } \\
\text { Teller, schlucke, schweige und sage } \\
\text { dann leise ohne Blickkontakt: «Na gut.» } \\
\text { Im Kiesler-Kreis wäre das wohl vor } \\
\text { allem submissiv. }\end{array}$ & $\begin{array}{l}\text { Sie haben hier sehr gut beschrieben, wie Sie sich } \\
\text { genau verhalten haben. Beim Kiesler-Kreis wäre } \\
\text { gegebenenfalls noch die Frage wichtig, ob durch } \\
\text { Ihr Verhalten Nähe oder Distanz zu Ihrer Frau } \\
\text { entstanden ist? Wenn eher Distanz, dann wäre } \\
\text { das Verhalten eher bei feindselig-submissiv } \\
\text { einzuordnen - was meinen Sie? }\end{array}$ & 1 \\
\hline 4. TE & $\begin{array}{l}\text { Ich gehe mit zum Sohn und den } \\
\text { Enkelkindern und danach zur Kantorei, } \\
\text { kann beides nicht genießen und habe } \\
\text { nachts extreme Schlafstörungen. }\end{array}$ & $\begin{array}{l}\text { Achtung, erinnern Sie sich an unsere Stunden? } \\
\text { Das tatsächliche Ergebnis sollte ja das Ende der } \\
\text { beschriebenen Situation enthalten und nicht } \\
\text { langfristige Konsequenzen. Der Trick besteht } \\
\text { doch darin, den letzten Satz aus Schritt } 1 \text { hier zu } \\
\text { übertragen, also in Ihrem Fall: Ich schweige kurz, } \\
\text { schlucke und sage: «Na gut.» Erinnern Sie sich? }\end{array}$ & 0 \\
\hline 5. EE & $\begin{array}{l}\text { Sie soll aufhören, mich so unter Druck } \\
\text { zu setzen. }\end{array}$ & $\begin{array}{l}\text { Auch hier tappen Sie in eine Falle, denn Ihr Ziel ist } \\
\text { zwar nachvollziehbar, jedoch Wunschdenken! } \\
\text { Beim EE ist es doch wichtig, Ziele zu finden, } \\
\text { die in Ihnen verankert sind und die Sie tatsächlich } \\
\text { ausführen können. Ein Vorschlag von mir: } \\
\text { «Ich sage meiner Frau, dass mir der Besuch bei } \\
\text { unserem Sohn und die Kantorei zu viel wird.» } \\
\text { Was halten Sie von diesem Vorschlag? } \\
\text { Oder wählen Sie einen anderen, aber Sie sollten } \\
\text { dabei aktiv werden, okay? }\end{array}$ & 0 \\
\hline 6. Vergleich TE und EE & Nein. & $\begin{array}{l}\text { Auch der Vergleich vom neuen TE und EE ergibt } \\
\text { ein: Nein! }\end{array}$ & - \\
\hline \multicolumn{4}{|l|}{ II. Lösungsphase } \\
\hline \multirow[t]{2}{*}{$\begin{array}{l}\text { 1. Revision der } \\
\text { Interpretationen }\end{array}$} & 1. Das war so. & $\begin{array}{l}\text { 1. Ja, diese Interpretation ist sehr gut und hilfreich } \\
\text { für das vorgeschlagene neue EE! }\end{array}$ & \\
\hline & $\begin{array}{l}\text { 2. Vielleicht eher: Ich glaube, dass ich } \\
\text { mich nicht wehren kann. } \\
\text { 3. Meine Frau soll mich endlich schonen } \\
\text { und nicht immer so unter Druck setzen. }\end{array}$ & $\begin{array}{l}\text { 2. Wie wäre es mit: «Ich habe gelernt, mich zu } \\
\text { wehren!» } \\
\text { 3. Wie finden Sie diesen Vorschlag: «Ich darf und } \\
\text { sollte nein sagen - das habe ich doch in Freiburg } \\
\text { gelernt!» }\end{array}$ & \\
\hline 2. Revision des Verhaltens & $\begin{array}{l}\text { Vielleicht hätte ich doch etwas anders } \\
\text { als «na gut» sagen sollen, aber ich traue } \\
\text { mir das noch nicht gegenüber meiner } \\
\text { Frau. }\end{array}$ & $\begin{array}{l}\text { Ja, Sie hätten - genauso wie Sie es so oft in } \\
\text { Freiburg gemacht haben - freundlich dominant } \\
\text { Ihrer Frau offen Ihre Bedenken schildern können. } \\
\text { Ich kann mir vorstellen, dass Ihre Frau dann mit } \\
\text { Ihnen diskutiert hätte und Sie einen Kompromiss } \\
\text { gefunden hätten, z.B. nur Kantorei oder nur } \\
\text { Besuch beim Sohn. Oder? }\end{array}$ & \\
\hline
\end{tabular}

(Fortsetzung nächste Seite) 
Tab. 1. (Fortsetzung)

\begin{tabular}{|c|c|c|}
\hline Schritte der SA & Herr Schmidt & Kommentar der Psychotherapeutin \\
\hline 3. Take-home-Message & Weiß ich nicht. & $\begin{array}{l}\text { Wie wäre es mit: «Ich sollte sagen, was ich denke! } \\
\text { Ich sollte für mich einstehen und meine } \\
\text { Bedürfnisse kommunizieren!» }\end{array}$ \\
\hline $\begin{array}{l}\text { 4. Generalisierung des } \\
\text { Gelernten }\end{array}$ & $\begin{array}{l}\text { Solche Situationen finden fast jeden Tag } \\
\text { statt - und nicht nur mit meiner Frau; } \\
\text { auch meine Kinder und selbst der } \\
\text { Nachbar will ständig etwas von mir. }\end{array}$ & $\begin{array}{l}\text { Dann können Sie dies auch auf die anderen } \\
\text { Situationen übertragen. }\end{array}$ \\
\hline $\begin{array}{l}\text { Allgemeine Anmerkungen } \\
\text { der Psychotherapeutin }\end{array}$ & $\begin{array}{l}\text { Vergessen Sie nicht, in Freiburg haben } \mathrm{Si} \\
\text { - denken Sie an die Konflikte mit dem eir } \\
\text { ob Sie ein langes Wochenende nach Haus } \\
\text { gut absagen können! Und ich bin mir sich } \\
\text { Rückfall haben, denn Sie merkt doch auc } \\
\text { letzten Paargespräch Ihre Frau ja auch vo } \\
\text { mich auf Ihre nächste SA! Bis dahin alles }\end{array}$ & $\begin{array}{l}\text { solch ähnliche Situationen oft schon so gut bearbeitet und gemeistert } \\
\text { n Mitpatienten oder auch damals die Anfrage von Ihrer Frau, } \\
\text { kommen, was für Sie da auch noch zu viel war. Da haben Sie doch } \\
\text {, dass Ihre Frau nicht gleich denken wird, dass Sie wieder einen } \\
\text { dass es Ihnen generell so viel besser geht. Zudem haben wir in dem } \\
\text { gereitet, dass Sie zunächst noch nicht voll belastbar sind. Ich freue } \\
\text { jute und viele liebe Grüße aus Freiburg! }\end{array}$ \\
\hline
\end{tabular}

während der Face-to-face-Therapie in der anschließenden Lösungsphase der SA im 1. Schritt mit dem Patienten, inwieweit die Interpretationen zutreffend und relevant sind und zum Ausgang der Situation beitragen. Sollten die Interpretationen für die Zielerreichung (EE) als hinderlich identifiziert werden, werden diese umstrukturiert. Häufig werden durch die SA Verhaltensdefizite aufgedeckt, die dann im 2. Schritt der Lösungsphase durch viele Rollenspiele mit «Shaping» verbessert werden. Bei chronisch depressiven Patienten spielt dabei häufig ein Training des Durchsetzungsvermögens eine wichtige Rolle. Manche Patienten müssen jedoch auch lernen, Kontrolle über reflexartige, feindselige Reaktionen $\mathrm{zu}$ bekommen und anstatt impulsiv zu reagieren, zunächst abzuwarten, wie sich die Situation entwickelt, um dann mit weniger Affekt überlegter reagieren zu können. Dies gilt insbesondere, wenn die emotionalen Ausbrüche eines Patienten seine Zielerreichung (EE) bisher behindert haben. In den beiden letzten Schritten der Lösungsphase wird der Patient motiviert, das in der SA Gelernte zusammenzufassen und auf ähnliche Situationen zu generalisieren, wodurch ein Lerntransfer hergestellt werden sollte.

Erste Studien unterstützen den klinischen Eindruck, dass die Leistung in der SA mit dem Behandlungserfolg in Zusammenhang zu stehen scheint: Studien zur ambulanten CBASPTherapie weisen darauf hin, dass die vom Therapeuten beurteilte Leistung (im Sinne des Beherrschens der Technik nach vorgegebenen Kriterien) in der SA im Verlauf der Therapie zunimmt, und dass diese Leistungssteigerung Prädiktor für die Reduktion der depressiven Symptomatik zum Therapieende zu sein scheint [Manber et al., 2003; Santiago et al., 2005; McCullough et al., 2010]. Dieser Befund konnte auch in einer stationären Pilotstudie repliziert werden: Patienten, die nach 6 Wochen Psychotherapie in der Lage waren, die SA effektiv einzusetzen, profitierten besonders gut von der stationären Behandlung. Außerdem erlitten diese Patienten nach Ab- schluss der stationären Therapie seltener Rückfälle (Brakemeier et al., 2013; Gutsgell, 2012, persönliche Mitteilungen). Auch diese Befunde sprechen dafür, Patienten beim nachhaltigen Einsatz der SA zu unterstützen. Um dieses Ziel zu erreichen, haben wir die im Folgenden beschriebene Nachsorgeintervention CBASP@home entwickelt.

Die Intervention CBASP@home ist auf einen 3-monatigen Behandlungszeitraum ausgelegt und beginnt nach der Entlassung aus der stationären Therapie. Die therapeutische Betreuung erfolgt durch einen in CBASP geschulten stationären Psychotherapeuten. Dieser soll möglichst der Einzeltherapeut sein, mit dem der Patient während des stationären Aufenthalts eine vertrauensvolle therapeutische Beziehung aufbauen kann. Während der ersten 6 Wochen findet die Intervention wöchentlich (= insgesamt 6 Termine), während der letzten 6 Wochen 2-wöchentlich (= insgesamt 3 Termine) statt. $\mathrm{Zu}$ jedem Termin füllen die Patienten das CBASP@home-Formular «Situationsanalyse» aus, das dem üblichen CBASP-Arbeitsblatt «Situationsanalyse» entspricht. Zusätzlich enthält es noch ein Feld, in dem die Psychotherapeuten zu jedem Schritt Feedback abgeben können. Ebenso können die Patienten konkrete Rückmeldung zu bestimmten Inhalten der SA vom Psychotherapeuten einfordern oder Fragen stellen bzw. Kommentare abgeben (Tab. 1 und 2).

Die Patienten erhalten innerhalb von 7 Tagen Rückmeldung zur SA durch den Psychotherapeuten. Bei der Rückmeldung wird der Psychotherapeut angehalten, zur Qualität eines jeden Schritts der SA Stellung zu nehmen. Hierbei wird er instruiert, zunächst positives Feedback zu geben, bevor gegebenenfalls auch Optimierungsoptionen thematisiert werden. Darüber hinaus beantwortet er die offenen Fragen oder Kommentare des Patienten. Insgesamt sollte das Feedback den Patienten motivieren, die SA weiterhin anzuwenden und ihn darin unterstützen, diese Strategie immer selbständiger und entsprechend den Kriterien durchzuführen. Der Psychothera- 
Tab. 2. Fallbeispiel Frau Rosenzweig: Eine SA aus der 7./8. Woche der CBASP@home-Intervention (PPRF-Leistung = 5)

\begin{tabular}{|c|c|c|c|}
\hline Schritte der SA & Frau Rosenzweig & Kommentar der Psychotherapeutin & PPRF-Wert \\
\hline \multicolumn{4}{|l|}{ I. Explorationsphase } \\
\hline 1. Situationsbeschreibung & $\begin{array}{l}\text { Ich stehe vor einem Café und sehe durch das } \\
\text { Fenster } 3 \text { Bekannte, die sich unterhalten. Ein } \\
\text { Stuhl ist frei. Ich betrete das Café, die Drei } \\
\text { schauen mich an. Ich sage: «Hallo Ihr Drei! } \\
\text { Kann ich mich zu Euch setzen?» }\end{array}$ & $\begin{array}{l}\text { Sehr schön! Sie haben alle Kriterien hier } \\
\text { genau beachtet! }\end{array}$ & 1 \\
\hline 2. Interpretation & $\begin{array}{l}\text { 1. Die kenne ich vom Spazierengehen. Das letzte } \\
\text { Mal haben wir uns nur sehr kurz unterhalten, } \\
\text { weil sie weg mussten. } \\
\text { 2. Ich mag sie und möchte wissen, wie es ihnen } \\
\text { geht. Zudem ist da ja auch noch ein Platz frei. } \\
\text { 3. Durchbrich die Mauer! Geh rein und sag } \\
\text { «hallo»! }\end{array}$ & $\begin{array}{l}\text { Super! Alle } 3 \text { Interpretationen sind in } \\
\text { der Situation verankert und helfen Ihnen, } \\
\text { Ihr EE zu erreichen! Sehr gut! }\end{array}$ & 1 \\
\hline 4. TE & $\begin{array}{l}\text { Ich betrete das Café und sage «Hallo Ihr Drei! } \\
\text { Kann ich mich zu Euch setzen?» }\end{array}$ & $\begin{array}{l}\text { Sehr gut - Sie übernehmen direkt den } \\
\text { letzten Satz aus dem } 1 \text {. Schritt. }\end{array}$ & 1 \\
\hline 5. EE & Ich betrete das Café und spreche die Drei an. & Genau! Super! & 1 \\
\hline 6. Vergleich TE und EE & $\mathrm{Ja} ! ! !+;$ & $\begin{array}{l}\text { Wie würde McCullough jetzt sagen: } \\
\text { «Let's have a party!» (; }\end{array}$ & - \\
\hline \multicolumn{4}{|l|}{ II. Lösungsphase } \\
\hline $\begin{array}{l}\text { 1. Revision der } \\
\text { Interpretationen }\end{array}$ & alle hilfreich und verankert & Ja, stimmt! & \\
\hline 2. Revision des Verhaltens & hilfreiches Verhalten & Auch das ist richtig! & \\
\hline 3. Take-home-Message & $\begin{array}{l}\text { Ich kann die Mauer überwinden und auf } \\
\text { Menschen zugehen! }\end{array}$ & Genau! & \\
\hline $\begin{array}{l}\text { 4. Generalisierung des } \\
\text { Gelernten }\end{array}$ & $\begin{array}{l}\text { Das kann ich auf viele Situationen übertragen, } \\
\text { sowohl privat als auch beruflich. }\end{array}$ & $\begin{array}{l}\text { Oh ja, das stimmt. Und ich bin mir recht } \\
\text { sicher, dass Sie das Gelernte nun wirklich } \\
\text { immer besser übertragen können! }\end{array}$ & \\
\hline Freie Kommentare & $\begin{array}{l}\text { Liebe Frau B., muss Ihnen unbedingt noch } \\
\text { berichten, wie es weiter ging. Ich habe mich dazu } \\
\text { gesetzt und die Drei haben sich echt gefreut. } \\
\text { Wir haben dann eine Stunde gemütlich Kaffee } \\
\text { getrunken und uns unterhalten. Am Ende haben } \\
\text { wir verabredet, in einer Woche uns in dem Café } \\
\text { wieder zu treffen! Toll, oder? }\end{array}$ & $\begin{array}{l}\text { Liebe Frau Rosenzweig, ich freue mich } \\
\text { wirklich sehr mit Ihnen über diesen } \\
\text { Erfolg! Das haben Sie wirklich ganz toll } \\
\text { gemacht, ich weiß ja, wie schwer Ihnen so } \\
\text { eine Situation vor der Therapie gefallen } \\
\text { wäre... Ihre Mauer haben Sie nun wirklich } \\
\text { (fast) überwunden - ganz toll, weiter so! }\end{array}$ & \\
\hline
\end{tabular}

peut sollte im Sinne des «Cheerleading» den Patienten motivieren, die CBASP-Strategien und insbesondere die SA zu Hause selbstständig weiter zu nutzen. Zudem findet bei jedem Termin ein Monitoring der depressiven Symptomatik mittels des Beck-Depressions-Inventars II statt (BDI-II) [Beck et al., 1996; Hautzinger et al., 1994]. Im Falle einer massiven Verschlechterung der Symptomatik oder bei Suizidalität nimmt der Psychotherapeut telefonischen Kontakt mit dem Patienten auf, um mit ihm eine Krisenintervention durchzuführen oder eine andere psychiatrische oder psychotherapeutische Intervention einzuleiten.

Um einen möglichst hohen Datenschutz zu gewährleisten, muss der Kontakt über einen Sicherheitsserver stattfinden. Der Zugang zur Kommunikationsplattform ist sowohl seitens der Patienten als auch der Psychotherapeuten durch Benut- zername und Passwort geschützt. Die Psychotherapeuten können die SA der Patienten nur innerhalb der Räumlichkeiten der betreffenden Klinik lesen und Rückmeldungen schreiben. Das CBASP@home-Programm, das von S.G. in Zusammenarbeit mit Bernd Tritschler (EDV) und E.L.B. entwickelt und programmiert wurde, ist bewusst einfach gestaltet, sodass diese Anwendung auch von weitgehend unerfahrenen Computernutzern bedient werden kann (vgl. Gutgsell, 2012, persönliche Mitteilung).

\section{Beschreibung von 2 Einzelfallanalysen}

Das Ziel der ersten Phase-I-Studie zu CBASP@home bestand darin, grundlegende Informationen zu sammeln, an- 
hand derer die einzelnen Interventionskomponenten und das Portal (technische Darbietung) der onlinebasierten CBASP-Intervention optimiert werden können. Anhand der beiden naturalistischen Einzelfallanalysen soll im Folgenden untersucht werden, ob die SA als Interventionskomponente tatsächlich umsetz- und durchführbar ist, und ob Anwendungsschwierigkeiten und/oder technische Probleme auftreten. Weiterhin wird untersucht, wie die depressive Symptomatik und die Fertigkeit des kriteriengerechten Durchführens der SA bei den beiden Patienten nach Entlassung aus der stationären Betreuung verlaufen, ob es zu Therapieabbrüchen kommt und wie zufrieden die Patienten mit der Intervention sind. Auf diese Weise sollen erste Erkenntnisse sowie Verbesserungsvorschläge für CBASP@home gewonnen werden.

\section{Ablauf und Durchführung der Pilotstudie zu CBASP@home}

Die CBASP-Hauptbehandlung (CBASP@5) wurde auf der Station 5 der Universitätsklinik Freiburg i.Br. durchgeführt [vgl. Brakemeier und Normann, 2012]. Voraussetzungen für die Teilnahme an CBASP@home waren eine vorangegangene stationäre Behandlung im Rahmen des CBASP@5-Konzepts, regelmäßiger Internetzugang, ausreichende Deutschkenntnisse und die Fähigkeit, Texte zu verfassen und zu lesen. Für die Rekrutierung kamen aus pragmatischen Gründen (Implementierung von CBASP@home im Rahmen einer Diplomarbeit von S.G.) insgesamt 6 Patienten in Frage. Diese wurden während ihres stationären Aufenthalts gefragt, ob sie an der Teilnahme an CBASP@home Interesse hätten und ausführlich über die Intervention, die Studie und die Datensicherheit informiert. Von den 6 Patienten entschlossen sich $4 \mathrm{zu}$ einer Teilnahme, die beiden anderen verzichteten wegen folgender Gründe auf die Teilnahme: Eine remittierte Patientin war der Ansicht, dass sie diese Behandlung nicht mehr benötige und zudem gut ambulant eingebunden sei, die andere Patientin hatte zusätzlich eine Aufmerksamkeits-Defizit-Hyperaktivitätsstörung, weshalb sie meinte, dass sie eine regelmäßige Teilnahme nicht gewährleisten könne und wolle. Von den 4 Patienten, die an CBASP@home teilnahmen, werden im Folgenden 2 Fälle ausführlich vorgestellt. Charakteristika der 4 teilnehmenden Patienten waren: keine Remission auf die stationäre Therapie (Responder oder Non-Responder), Angst vor der Entlassung und keine weitere CBASP-Therapie im Anschluss.

Nachdem die Patienten eingewilligt hatten, fand im Anschluss eine Einweisung in die Bedienung des Portals statt und die Patienten erhielten ein Handout, in dem alle besprochenen Informationen schriftlich zusammengefasst waren.

Die Erfassung der depressiven Symptomatik im Fremdurteil erfolgte mit Hilfe der 24-Item-Version der Hamilton-Depressionsskala (HAMD-24) [Hamilton, 1960, 1967]. Dabei wurde die Prä-Messung für CBASP@home im Rahmen der Abschlussmessung der stationären Therapie, maximal 14 Tage vor der ersten CBASP@home Behandlungswoche und maximal 7 Tage nach Ablauf der regulären Behandlungszeit erhoben. Die Post-Messung erfolgte am Ende der letzten CBASP@home-Woche durch ein telefonisches Interview. Die Prä-, Post- und Verlaufsmessungen der depressiven Symptomatik im Selbsturteil fanden während CBASP@home mittels BDI-II via Onlineplattform statt. Die Remission wurde als ein HAMD-24-Wert von $\leq 10$ bzw. BDI-II-Wert $\leq 10$ Punkten und Response als Reduktion der depressiven Symptomatik um $\geq 50 \%$ definiert [vgl. Beck et al., 1996; Brakemeier et al., 2012a; Zimmermann et al., 2004].

Die Leistung in der SA wurde anhand der Patient Performance Rating Form (PPRF) erfasst [McCullough, 2010, 2000; deutsch: Schramm et al., 2006]. Die Leistung umschreibt hier das Ausmaß, in dem der Patient in der Lage ist, die SA nach vorgegebenen Kriterien selbständig (d.h. ohne Unterstützung durch den Psychotherapeuten) durchzuführen. Der Fragebogen wird im Fremdurteil durch den Psychotherapeuten im Anschluss an eine durchgeführte SA ausgefüllt. Bei jedem der 5 Schritte der Explorationsphase (siehe oben) wird jeweils dichotom bewertet, ob der Patient diesen Schritt entsprechend den Kriterien ausgeführt hat oder nicht. Somit können minimal 0 und maximal 5 Punkte erreicht werden. Bislang liegen noch keine Gütekriterien der PPRF vor. Erste Analysen der Beurteilungsübereinstimmung der PPRF legen für den Gesamtwert eine «akzeptable» bis «gute» Übereinstimmung nahe [vgl. McCullough, 2000, 2010].

Der Fragebogen zur Zufriedenheit mit und Umsetzbarkeit von CBASP@home (Gutgsell und Brakemeier, 2012, persönliche Mitteilung) umfasst insgesamt 22 Items, wobei 8 Items dem Fragbogen zur «Erfassung der Patientenzufriedenheit der E-Mail-Brücke» [Wolf et al., 2006, 2008] mit freundlicher Genehmigung entnommen wurden. Auf einer 5-stufigen Skala von «überhaupt nicht» bis «sehr» wurde die Zufriedenheit mit bzw. die subjektive Nützlichkeit der Intervention durch folgende Items erhoben: «Insgesamt bin ich mit CBASP@home zufrieden»; «Das Schreiben von SA hat mir geholfen»; «CBASP@home als Brücke zwischen stationärer Behandlung und Alltag halte ich für sinnvoll»; «CBASP@ home hat mir geholfen, die Technik der SA in den Alltag zu übertragen». Darüber hinaus wurden jeweils 4 Fragen zur technischen Umsetzung (z.B. «Das Programm war leicht zu bedienen»; «Ich hatte technische Probleme mit dem CBASP@home-Portal»), zur Dauer und Frequenz der Intervention (z.B. «3 Monate Teilnahmedauer fand ich ausreichend»; «Ein Termin pro Woche in den ersten 6 Behandlungswochen war ausreichend») und zur Auswahl des Psychotherapeuten (z.B. «Es ist wichtig und sinnvoll, dass der stationäre Einzeltherapeut die Betreuung durchführt»; «Eine Betreuung durch einen CBASP@5-Therapeuten, den ich nicht persönlich kenne, ist genauso sinnvoll und hilfreich») gestellt. Die Einzelfallbeschreibungen wurden deskriptiv ausgewertet, wobei die Berechnungen der Summenwerte mittels Excel erfolgten. 
Tab. 3. Soziodemographische und klinische Merkmale der beiden CBASP@home-Einzelfälle

\begin{tabular}{|c|c|c|}
\hline Fallbeispiel-Patienten & Herr Schmidt & Frau Rosenzweig \\
\hline Alter & 69 Jahre & 46 Jahre \\
\hline Demographische Daten & verheiratet, 3 Söhne, Abitur & geschieden, 1 Sohn, Hauptschulabschluss \\
\hline Unmittelbar vorausgegangene Behandlung & CBASP@5-Hauptbehandlung & CBASP-Fresh-up (Hauptbehandlung 12 Monate zuvor) \\
\hline Hauptdiagnose & $\begin{array}{l}\text { rezidivierende depressive Störung, } \\
\text { aktuell schwere depressive Episode } \\
\text { ohne psychotische Symptome, zudem } \\
\text { Dysthymie (ICD-10: F33.2, F34.1) }\end{array}$ & $\begin{array}{l}\text { rezidivierende depressive Störung, aktuell schwere } \\
\text { depressive Episode ohne psychotische Symptome, } \\
\text { zudem Dysthymie (ICD-10: F33,2, F34,1) }\end{array}$ \\
\hline Dauer der aktuellen Episode & 44 Monate & $\begin{array}{l}134 \text { Monate (vor CBASP-Hauptbehandlung } \\
+12 \text { Monate) }\end{array}$ \\
\hline Alter bei Erkrankungsbeginn & 46 Jahre & 8 Jahre \\
\hline Anzahl der Episoden & 4 Episoden & 3 Episoden \\
\hline Ausgewählte komorbide Störungen & $\begin{array}{l}\text { schädlicher Gebrauch von } \\
\text { Benzodiazepinen (ICD-10: F13.1), } \\
\text { zwanghafte Persönlichkeitsstörung, } \\
\text { Morbus Parkinson }\end{array}$ & $\begin{array}{l}\text { selbstunsicher-vermeidende Persönlichkeitsstörung, } \\
\text { Myoklonus-Dystonie-Syndrom, chronisches } \\
\text { Schmerzsyndrom (Hals- und Brustwirbelsäulensnydrom } \\
\text { (HWS/BWS), multiple Gelenke), Migräne }\end{array}$ \\
\hline $\begin{array}{l}\text { Leistung in der SA (PPRF) während des } \\
\text { stationären Aufenthalts }\end{array}$ & prästationär: 1 , poststationär: 3 & prästationär: 5 , poststationär: 5 \\
\hline $\begin{array}{l}\text { Depressive Symptomatik (HAMD-24) } \\
\text { stationär / prä und post }\end{array}$ & prästationär: 26; poststationär: 13 & prästationär: 21; poststationär: 21 \\
\hline Ansprechstatus & Response auf die stationäre Behandlung & Non-Response auf die stationäre Behandlung \\
\hline $\begin{array}{l}\text { Parallele therapeutische Behandlung zu } \\
\text { CBASP@home }\end{array}$ & $\begin{array}{l}\text { tagesklinische Betreuung während } \\
3 \text { Wochen (keine Psychotherapie) }\end{array}$ & $\begin{array}{l}\text { ambulante Psychotherapie (keine CBASP- } \\
\text { Therapie) }\end{array}$ \\
\hline
\end{tabular}

PPRF = Patient Performance Rating Form (Skala 1-5), HAMD = Hamilton Depression Rating Form (Skala 0-78).

\section{Falldarstellungen}

Von den 4 an der Pilotstudie teilnehmenden Patienten werden im Folgenden 2 Fälle skizziert, um exemplarisch die Umsetz- und Durchführbarkeit sowie die Akzeptanz und den subjektive Nutzen der Intervention zu beschreiben. Für diesen Zweck wählten wir einen männlichen 69-jährigen Patienten (Herrn Schmidt) und eine weibliche 46-jährige Patientin (Frau Rosenzweig; Patientennamen geändert), die die Kriterien einer chronischen Depression erfüllten und die bereits beschriebenen Merkmale einer chronischen Depression aufwiesen. Bei Frau Rosenzweig lag der für chronifizierte depressive Störungen typische frühe Erkrankungsbeginn mit Traumatisierung durch emotionale Vernachlässigung und Missbrauch vor. Die derzeitige depressive Episode hielt bereits 11,2 Jahre an, wobei dies die 3. Episode war. Herr Schmidt hatte einen late-onset (Ersterkrankung mit 46 Jahren), wobei er 3,8 Jahre an der derzeitigen Episode litt und zuvor bereits 4 schwere Episoden erlebt hatte. Beide Patienten litten unter mehreren komorbiden Erkrankungen und wiesen beide das gleichzeitige Bestehen einer Dysthymie und einer rezidivierenden depressiven Störung auf. Bei Herrn Schmidt lagen zusätzlich ein schädlicher Gebrauch von Benzodiazepinen sowie eine zwanghafte Persönlichkeitsstörung vor. Frau Rosenzweig erfüllte die Kriterien einer selbstunsicher-vermeidenden Persönlichkeitsstörung. Zudem hatte sie verschiedene körperliche Erkrankungen und Beeinträchtigungen (Tab. 3).

Herr Schmidt nahm unmittelbar vor CBASP@home an der 3-monatigen stationären Hauptbehandlung im Rahmen des CBASP@5-Konzepts teil und wurde als Responder (HAMD-24 bei Aufnahme = 26, bei Entlassung $=13$ ) aus der stationären Behandlung entlassen. Da er aus einem kleinen Dorf in Bayern kam, wo es im näheren Umkreis keine in CBASP geschulten Psychotherapeuten gab und er große Angst hatte, nach Entlassung wieder einen Rückfall zu erleiden, nahm er das CBASP@home Angebot sehr gerne wahr. Zusätzlich wurde für ihn ein tagesklinischer Behandlungsplatz in einer nahegelegenen Versorgungsklinik organisiert, an der er unterstützende Gespräche (keine Psychotherapie) sowie medi- kamentöse Optimierung und Tagesstruktur durch weitere Fachtherapien erhielt. Während des stationären Aufenthalts zeigte Herr Schmidt einen Lernzuwachs in der therapeutenbeurteilten Leistung der SA vom Präzum Post-Messzeitpunkt (PPRF Prä-Post-Differenz: 2 Punkte), erreichte aber nicht die volle Punktzahl (Tab. 3).

Bei Frau Rosenzweig fand diese CBASP-Hauptbehandlung ein Jahr zuvor statt. Damals sprach sie als Responderin auf die Behandlung an. Sie nahm vor CBASP@home aufgrund eines Rückfalls in die Depression an einer 1-monatigen CBASP Fresh-up-Behandlung teil, was Teil des Konzepts ist [Brakemeier und Normann, 2012]. Auf die Fresh-up-Behandlung sprach sie leider nicht an (HAMD-24 bei Aufnahme $=21$, bei Entlassung = 21), gab aber an, dass ihr die CBASP-Strategien sehr helfen würden. Der Aufenthalt sei jedoch viel zu kurz für all ihre derzeitigen Probleme gewesen. Daher zeigte sie sich besonders motiviert, an CBASP@home teilzunehmen. Parallel zu CBASP@home nahm Frau Rosenzweig alle 2 Monate Termine bei ihrer Psychotherapeutin war, bei der sie schon lange in verhaltenstherapeutischer Behandlung war, die jedoch nicht in CBASP geschult war. Bezüglich der Leistung in der SA begann Frau Rosenzweig das Fresh-up bereits auf höchstem Niveau, das über alle 3 Messzeitpunkte hinweg konstant blieb. Einen Überblick über die klinischen und soziodemographischen Daten der beiden Patienten gibt Tabelle 3.

\section{Ergebnisse der Fallbeispiele}

\section{Ergebnisse zur Zufriedenheit mit und Umsetzbarkeit von}

\section{CBASP@home}

Fehlzeiten und Abbrüche: Als ein Indikator für die Zufriedenheit mit der Intervention wird die Anzahl der wahrgenommenen Termine betrachtet. Beide Patienten beendeten CBASP@home wie vorgesehen nach 3 Monaten. Während Herr Schmidt alle 9 Termine sehr gerne in Anspruch nahm, nutzte Frau Rosenzweig einen Termin nicht, da ihr «keine Situation eingefallen» sei. 
Abb. 2. Verlauf der therapeutenbeurteilten Leistung in der SA (PPRF) und der depressiven Symptomatik im Selbsturteil (BDI-II). PPRF= Patient Performance Rating Form (Skala 1-5, ein Intervall entspricht einem PPRFPunkt); BDI II = Beck-DepressionsInventar II (Skala 0-63, hier abgebildet $0-30$, ein Intervall entsprich 5 BDI-IIPunkten). w46: Fallbeispiel Frau Rosenzweig; m69: Fallbeispiel Herr Schmidt.

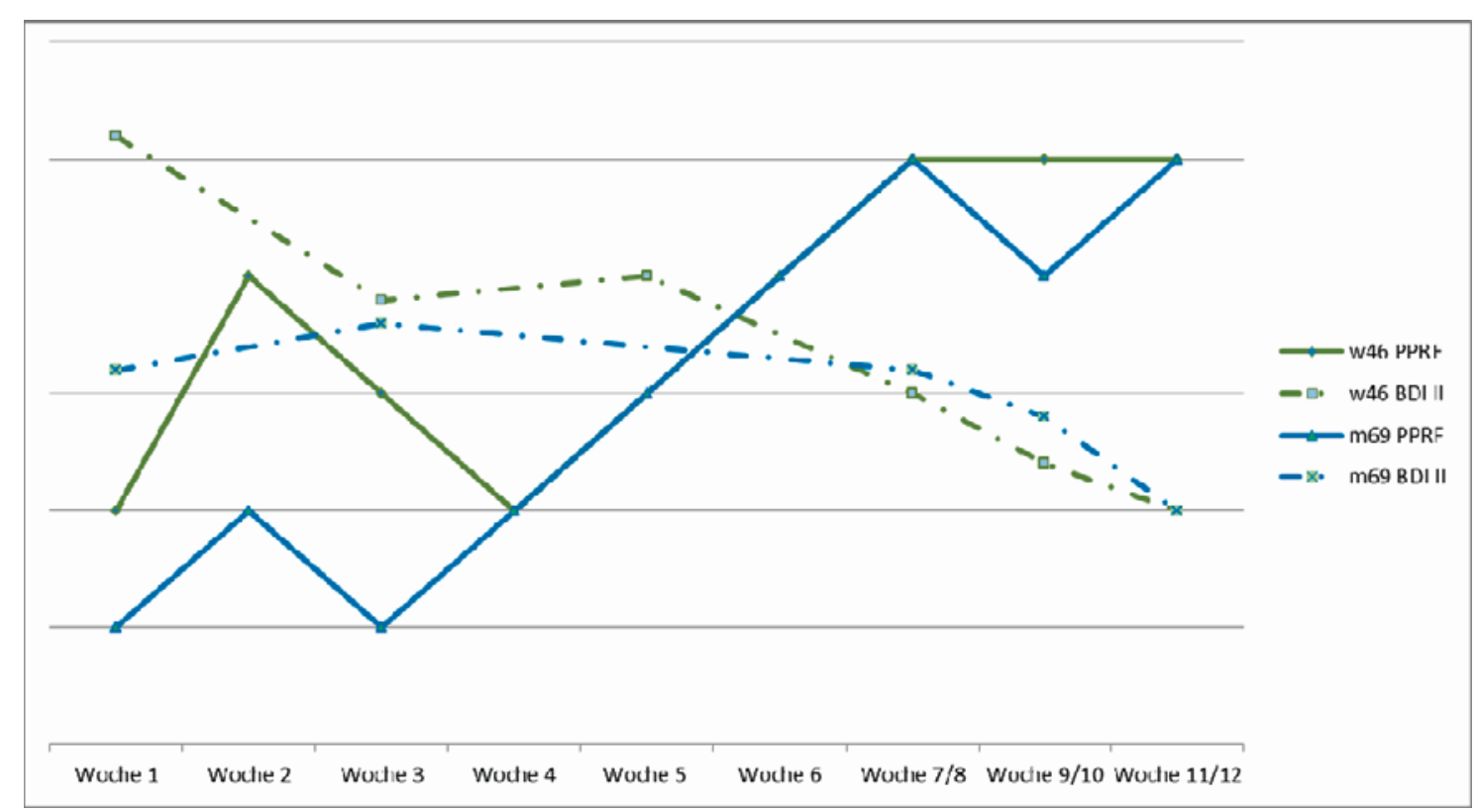

Tab. 4. Selbst- und fremdbeurteilte Depression zum Prä- und PostMesszeitpunkt der CBASP@home-Pilotstudie

\begin{tabular}{lll}
\hline & Herr Schmidt & Frau Rosenzweig \\
\hline HAMD-24 & & \\
CBASP@home / prä* & 13 (Remission) & 21 (Remission) \\
CABSP@home / post & 10 & 6 \\
BDI-II & & 16 \\
CBASP@5 poststationär & 19 & 26 \\
CBASP@home / prä & 16 & 10 (Remission) \\
CBASP@home / post & 10 (Remission) & \\
\hline *Entspricht dem CBASP@5-post-Wert. & \\
\hline
\end{tabular}

Ergebnisse der Fragebogenevaluation: Die Zufriedenheit mit CBASP@home wurde durch den Fragebogen zur Zufriedenheit mit und Umsetzbarkeit von CBASP@home ermittelt [Wolf et al., 2008]. Die Intervention sowie deren technische und personelle Umsetzung stießen bei beiden Patienten auf hohe Akzeptanz und Zufriedenheit. Beispielsweise bewerteten beide Patienten das Schreiben der SA als «ziemlich» oder «sehr» hilfreich und waren in gleichem Maße zufrieden mit der Intervention. Beide Patienten empfanden CBASP@home als «ziemlich»oder «sehr» hilfreiche Brücke zwischen stationärer Behandlung und dem Alltag.

Die Dauer der Intervention wurde von Herrn Schmidt als «etwas» und von Frau Rosenzweig als «sehr» zufriedenstellend bewertet. Einen Therapietermin pro Woche während der ersten 6 Wochen empfanden beide Patienten als «sehr» ausreichend. Frau Rosenzweig stufte einen Therapietermin alle 2 Wochen als «sehr» ausreichend, Herr Schmidt als «ziemlich» ausreichend ein. Das Warten auf eine Rückmeldung zur SA wurde von Frau Rosenzweig als «überhaupt nicht» zu lang empfunden, Herr Schmidt empfand das Warten als «ein wenig» zu lang.

Auch die Bewertungen der technischen Umsetzung vielen positiv aus. Beide Patienten empfanden das Programm als «ziemlich» leicht zu bedienen. Bei Herrn Schmidt seien während der Nutzung des Portals «überhaupt keine» technischen Probleme aufgetreten, Frau Rosenzweig gab an, «wenig» technische Probleme gehabt zu haben. Beiden Patienten erschien das Onlineformular zur Erstellung der SA «ziemlich» oder «sehr» sinnvoll gegliedert und übersichtlich gestaltet zu sein.
Die Rolle des Psychotherapeuten im Rahmen des CBASP@homeKonzepts wurde von beiden Patienten als «ziemlich» wichtig empfunden. Außerdem empfanden es beide Patienten als «ziemlich» (Frau Rosenzweig) oder «sehr» (Herr Schmidt) wichtig und sinnvoll, dass der stationäre Einzeltherapeut die CBASP@home-Betreuung übernahm. Die Anschlussbetreuung durch einen anderen, ihnen aus der stationären Therapie bekannten und ebenfalls in CBASP geschulten Psychotherapeuten, erschien den Patienten ebenfalls «ziemlich» sinnvoll zu sein. Die Option, von einem Psychotherapeuten betreut zu werden, den sie nicht kennen, wurde von beiden Patienten hingegen als «überhaupt nicht» hilfreich bewertet.

Die beiden Psychotherapeutinnen, welche die beiden Patienten im Rahmen von CBASP@home betreut hatten, gaben an, im Durchschnitt 18 min für ein Feedback zur SA benötigt zu haben (Range: 9-31 min), wobei die zeitliche Belastung im Verlauf immer geringer wurde. Dies ist darauf zurückzuführen, dass die Patienten die SA immer besser/kriteriengerechter durchführten (Tab. 3, Abb. 2).

Veränderung der Leistung in der SA und der depressiven Symptomatik

Die erste SA von Herrn Schmidt im Rahmen von CBASP@home überraschte seine Psychotherapeutin, da er nahezu alle Kriterien nicht beachtet hatte und so einen PPRF-Wert von 1 erzielte. Beim Feedback lobte sie zunächst die Tatsache, dass er die SA überhaupt geschickt habe, gab ihm jedoch klares Feedback zu jedem Schritt und erinnerte ihn an die relevanten Kriterien. In Tabelle 1 ist beispielhaft die 2. SA von Herrn Schmidt einschließlich der Kommentare seiner Psychotherapeutin dargestellt. In der letzten Spalte finden sich auch die PPRF-Werte, zu denen Herr Schmidt jedoch keine Rückmeldung erhalten hat.

Die PPRF-Leistung der folgenden 3 SA von Herrn Schmidt war noch relativ schwach, wobei er immer wieder dankbar das kritische Feedback seiner Psychotherapeutin aufnahm. In Folge des Feedbacks gelang es ihm jedoch im Verlauf, die verbliebenen SA immer besser zu bearbeiten, sodass er am Ende die Höchstpunktzahl erreichen konnte (Abb. 2). Bei den letzten 3 SA erreichte er stets seine Ziele, die nun in ihm verankert und realistisch umsetzbar waren. Er hatte gelernt, seine Bedürfnisse auch zu Hause gegenüber seiner Frau und anderen Angehörigen sowie Freunden zu kommunizieren, sich abzugrenzen und auch durchzusetzen. Am Ende berichtete er, dass durch sein neues Verhalten wieder mehr emotionale Nähe zu seiner Frau entstanden sei, was beide sehr genießen würden. Sie würde mehr Rücksicht auf ihn nehmen und ihn für kleine Schritte sehr loben. 
Auch Frau Rosenzweig hatte zu Hause zunächst einen Leistungseinbruch bezüglich der SA. Mit Hilfe des Feedbacks Ihrer Psychotherapeutin schaffte sie es jedoch ebenfalls, am Ende von CBASP@home eigenständig eine «perfekte» SA zu erstellen (Tab. 2).

Abbildung 2 stellt den Zusammenhang zwischen der therapeutenbeurteilten Leistung in der SA und der Veränderung der depressiven Symptomatik im Selbsturteil während des Verlaufs von CBASP@home dar [vgl. McCullough et al., 2010]. Eine hohe PPRF-Punktzahl ist dabei gleichzuset zen mit einer besseren Leistung in der SA, eine niedrige BDI-II-Punktezahl gleichzusetzen mit einer geringeren depressiven Symptomatik. Die Graphik verdeutlicht bei beiden Patienten eine Prä-/Post-Zunahme (Beginn vs. Ende der Therapie) der Leistung im kriteriengerechten Erstellen der SA. Auffällig ist gleichzeitig eine temporäre Verschlechterung der Leistung im gesamten Therapieverlauf, denn bei beiden Patienten lag die Leistung zu Beginn von CBASP@home unter ihrer Leistung zum Ende der Hauptbehandlung (Tab. 3). Frau Rosenzweigs Leistung verschlechterte sich in dieser Zeit vom höchstmöglichen Wert um -3 Punkte. Bei Herrn Schmidt lag die Differenz zwischen Ende der Hauptbehandlung und Beginn der Nachsorgeintervention bei -2 Leistungspunkten. Zudem kam es im Verlauf von CBASP@home bei beiden Patienten zu einem vorübergehenden Leistungsabfall bezüglich der SA. Zum Schluss waren jedoch beide in der Lage, die SA «perfekt», d.h. kriteriengerecht durchzuführen, was sich im Erreichen der vollen Punktzahl (PPRF = 5) ausdrückte (Abb. 2, Tab. 3).

Bezüglich der Depressivität hatte die Fresh-up-Patientin im Selbsturteil (BDI-II) in den 2-3 Wochen zwischen der stationären Entlassung und dem Start von CBASP@home eine Symptomverschlechterung erfahren (BDI-II: CBASP@5 post = 16, CBASP@home prä = 26). Trotzdem erreichte sie nach CBASP@home die Remission (BDI-II: CBASP@home post =10). Auch Herr Schmidt konnte am Ende von CBASP@home als Remitter klassifiziert werden (BDI-II: CBASP@5 post=19, CBASP@ home prä=16, CBASP@home post =10). Demensprechend zeigt sich in Abbildung 2 ein Anstieg der SA bei beiden Patienten, deren Werte sich mit denjenigen der abfallenden BDI-II-Werte kreuzen.

Auch im Fremdurteil (HAMD-24) konnten beide Patienten das Remissionskriterium am Ende von CBASP@home erreichen: Herr Schmidt hatte zum CBASP@home Prä-Messzeitpunkt 13 Punkte und zum PostMesszeitpunt 10 Punkte auf der HAMD-24-Skala erreicht. Frau Rosenzweig hatte auf der HAMD-24-Skala zum CBASP@home Prä-Messzeitpunkt 21 Punkte und zum Post-Messzeitpunt 6 Punkte erzielt.

\section{Diskussion}

Mit Hilfe von 2 Fallbeschreibungen sollen erste Erkenntnisse über CBASP@home gewonnen werden. Hierfür wurde ermittelt, wie zufrieden die beiden Patienten mit der Intervention, der technischen Umsetzung und der Wahl des Psychotherapeuten waren. Zudem wurde der Verlauf der therapeutenbeurteilten Leistung in der SA und der depressiven Symptomatik beschrieben.

Die Zufriedenheit mit der Intervention wurde von beiden Patienten als hoch bewertet, wobei die Patienten die Intervention für eine sinnvolle Möglichkeit der Überbrückung der Übergangsphase zwischen stationärem Aufenthalt und Alltag empfunden haben. Der Umstand, dass es bei den beiden beschriebenen Patienten keinen Therapieabbruch gab und insgesamt nur ein Termin nicht genutzt wurde, deutet ebenfalls auf eine hohe Akzeptanz der Intervention hin.

Da die 2 beschrieben Einzelfälle jedoch nicht zwangsläufig repräsentativ sind, ist auch nicht auszuschließen, dass beispiels- weise die Urteile zur Zufriedenheit bei Patienten ohne Ansprechen auf die Intervention oder mit Rückfall während der Intervention weniger positiv ausfallen können. Beide Patienten remittierten im Verlauf von CBASP@home, was sich wahrscheinlich auch in den durchweg positiven Bewertungen ausdrückt. Es erscheint logisch, dass der Verlauf der depressiven Symptomatik während der Nachsorgeintervention im $\mathrm{Zu}$ sammenhang mit der Zufriedenheit der Patienten steht. Für nachfolgende Untersuchungen wäre es daher spannend zu prüfen, ob sich die hohe Zufriedenheit mit CBASP@home auch bei Patienten mit weniger günstigen Symptomverläufen zeigt.

Die Zufriedenheit der Teilnehmer und der Rückgang der depressiven Symptomatik lässt auf die Durchführbarkeit, Akzeptanz und Effektivität der Intervention hoffen. Insbesondere unter Beachtung, dass es sich bei den eingeschlossenen Einzelfällen um langjährige chronifizierte und komorbid belastete Patienten mit mittlerem bis hohem Lebensalter handelte, die teilweise sehr geringe Bildung sowie Computerkenntnisse besaßen, erscheint CBASP@home als vielversprechende Möglichkeit der Stabilisierung und Rückfallprävention. In einer randomisierten Studie mit 400 Patienten, die ebenfalls eine internetbasierte Erhaltungstherapie nach stationärem Aufenthalt untersuchte, konnte niedriges Bildungsalter als ein positiver Prädiktor für den Erfolg der Onlinenachsorge identifiziert werden [Ebert et al., 2013a]. Möglicherweise haben Patienten mit niedrigem Bildungsalter und daraus resultierend geringen Computervorkenntnissen mehr Mühe, in die Regelversorgung zu gelangen, weshalb sie von speziellen internetbasierten Maßnahmen besonders profitieren.

Mit der Umsetzung der CBASP@home-Intervention waren die beiden Patienten überwiegend zufrieden. Bezüglich der Dauer der Intervention gingen die Meinungen leicht auseinander. Der Wunsch von Herrn Schmidt nach einer Verlängerung der Intervention - obwohl er bezüglich der Depression remittiert war - lässt sich vor dem Hintergrund gut verstehen, dass er als Einwohner eines abgeschiedenen Orts ohne psychologische Nachbetreuung im ambulanten Setting auskommen musste. Vor der CBASP-Therapie hatte er ein sehr medizinisches Verständnis der Depression und war der Meinung, dass nur Medikamente oder die Elektrokonvulsionstherapie ihm helfen könnten. Nach CBASP war er jedoch der Überzeugung, dass speziell die CBASP-Strategien für ihn hilfreich sind, war sich jedoch noch unsicher, ob er diese eigenständig weiter nutzen könne. Für solche Patienten scheint eine Onlinenachbetreuung wie CBASP@home ideal. Für Frau Rosenzweig war die 3-monatige Dauer hingegen ausreichend - vermutlich weil sie danach auch weiter psychotherapeutisch angebunden war. Sie erzählte am Ende über CBASP@home, dass sie nun ihrer Verhaltenstherapeutin die SA beibringen wolle.

Das unterschiedliche Bedürfnis nach einer Verlängerung der Intervention deckt sich mit einer der Phase-I-Studie vorangegangenen Befragung von 33 ehemaligen stationären CBASP@5-Patienten. Hier wünschten sich 12,1\% der Patien- 
ten weniger als 3 Monate, 12,1\% 3 Monate und 75,8\% mehr als 3 Monate (Minimum $=0$; Maximum = 18; Mittelwert $(\mathrm{MW})=6,68$; Standardabweichung $(\mathrm{SD})=4,19 ;$ Median $(\mathrm{Med})=6,0)$ Nachbetreuung (online oder Telefon; Gutgsell, 2012, persönliche Mitteilung). Auch die Evaluation der EMail-Brücke [Wolf et al., 2006, 2008], der Internet-Chatgruppe [Kordy et al., 2006] sowie einer 12-wöchigen transdiagnostischen webbasierten Rehabilitationsnachsorge (W-RENA) [Ebert et al., 2013a] erbrachte vergleichbare Ergebnisse. Möglicherweise fürchten Patienten generell, nach Psychotherapien erzielte Behandlungserfolge nicht eigenständig aufrechterhalten zu können. Bei chronisch depressiven Patienten mag diese Befürchtung aufgrund ihrer bisherigen Erfahrungen (sehr lange Episodendauer und gehäuft Rückfälle) besonders ausgeprägt zu sein. Diese Vermutung ist allerdings spekulativer Natur, da die genannten Studien die Nachsorgewünsche in heterogenen klinischen Stichproben erfassten [Wolf et al., 2006, 2008; Kordy et al., 2006; Ebert et al., 2013a] und keine Subgruppenanalysen speziell für chronisch depressive Patienten durchgeführt wurden.

Auf der Basis der beschriebenen Forschungsergebnisse ließe sich vermuten, dass es auch innerhalb einer größeren Evaluationsstichprobe von CBASP@home zu vergleichbar divergierenden Wünschen bezüglich der Länge der Intervention gekommen wäre. Inwieweit den Patientenbedürfnissen nach einer längeren Teilnahmedauer nachgegangenen werden kann und sollte, ist jedoch fraglich. Zum einen ist dies nicht im Einklang mit dem angedachten Konzept der Intervention als vorübergehende Stütze und Begleitung in den Alltag, zum anderen sind solchen Vorhaben auch finanzielle und personelle Grenzen gesetzt. Es wäre wünschenswert, dass zukünftige Studien sowohl die Dauer der Intervention, als auch die Sitzungsfrequenz variieren, damit diesbezüglich evidenzbasierte Empfehlungen abgegeben werden können.

Positiv verlief die Bewertung der technischen Umsetzung. Hier wählten beide Patienten das Attribut «ziemlich» leicht $\mathrm{zu}$ bedienen. Auch traten überhaupt keine oder nur wenige technische Probleme auf, die ausschließlich mit anfänglichen Programmierungsfehlern in Zusammenhang standen. Die Einstufung des CBASP@home-Portals ist umso erstaunlicher, als beide Patienten zuvor angaben, unerfahren im Umgang mit dem Computer zu sein. Ähnliche Befunde wurden auch in anderen Studien beschrieben [Ebert et al., 2013a; Lin et al., 2013]. Möglicherweise ist eine Einweisung bzw. Schulung hinsichtlich derartiger internetbasierter Nachsorgeprogramme während des stationären Aufenthalts der entscheidende Vorteil gegenüber internetbasierten Therapien, die ohne vorherige stationäre Behandlung beginnen. Die bewusst einfach gehaltene Gestaltung hat sich somit bewährt und sollte künftig beibehalten werden. Das Onlineformular zur SA erschien beiden Patienten als ziemlich bis sehr sinnvoll gegliedert und übersichtlich gestaltet.

Das Warten auf die Rückmeldung des Psychotherapeuten wurde von wenig bis überhaupt nicht zu lang eingestuft. Aus klinischer Sicht wäre es dennoch sinnvoll, wenn die Rückmeldungen künftig zeitnaher erfolgen. Dies würde zum einen ein rechtzeitiges Entdecken von Krisen und das Einleiten geeigneter Interventionen ermöglichen. Zum anderen ist gegebenenfalls auch der Lerneffekt größer, wenn eine Verstärkung unmittelbarer erfolgt und Fehler schneller korrigiert werden. Um dies in künftigen Studien zu ermöglichen, müssten jedoch die Personalressourcen erweitert werden.

Generell scheint der Psychotherapeut für die Patienten eine bedeutende Rolle im Rahmen des CBASP@home-Konzepts einzunehmen. Die Patienten erachteten es als ziemlich bzw. sehr wichtig und sinnvoll, dass der stationäre Einzeltherapeut auch die CBASP@home-Betreuung durchführt. Die Onlinebetreuung durch einen anderen, bereits von der stationären Therapie bekannten Psychotherapeuten wurde als ziemlich sinnvoll bewertet. Eine Betreuung durch einen ihnen unbekannten Psychotherapeuten erschien beiden Patienten überhaupt nicht sinnvoll. Diese Bewertungen unterstützen das vorgestellte Step-down-Konzept, in dem aufbauend auf einer bestehenden therapeutischen Beziehung eine nahtlose Weiterbetreuung angeboten werden soll. Da zur Heilung früherer traumatisierender Beziehungserfahrungen insbesondere der Beziehungsgestaltung durch DPE und IDÜ eine bedeutsame Rolle eingeräumt wird, erscheint es sehr nachvollziehbar, dass die Patienten weiter von ihrem Psychotherapeuten betreut werden möchten.

Bei der Implementierung einer derartigen Onlinenachsorge ist es natürlich auch relevant zu erfahren, wie die beteiligten Psychotherapeuten die Behandlung hinsichtlich Umsetzbarkeit, Sinnhaftigkeit und Wirksamkeit beurteilen. Die beiden beteiligten Psychotherapeutinnen (E.L.B., V.E.) empfanden den zusätzlichen Aufwand, den sie im Rahmen ihrer regulären Arbeitszeit ableisten mussten, gerade jeweils in den ersten Wochen von CBASP@home als hoch, wobei der Aufwand jeweils im Verlauf der Behandlung eines jeden Patienten weniger und damit machbarer wurde. Sinnvoll erscheint bei derartigen Programmen in jedem Fall die Schaffung zusätzlicher Kapazitäten, sodass CBASP@home in größerem Umfang mit zeitnaher Rückmeldung ohne zusätzliche Belastung der Psychotherapeuten umsetzbar ist. Beide Psychotherapeutinnen stimmten überein, dass CBASP@home eine sehr sinnvolle und auch wirksame Nachbehandlung ist, wenn denn die Patienten ausreichend motiviert sind, die SA regelmäßig zu erstellen. Zudem berichteten beide, dass die Rückmeldung auch Freude bereite und es eine sehr schöne Art sei, nach einer intensiven Behandlung weiter mit den Patienten in Kontakt zu sein. Jedoch empfanden beide es auch als sinnvoll, die Patienten mit ihren Prägungen und spezifischen Schwierigkeiten sowie Ressourcen bereits aus der stationären Behandlung gut zu kennen. Es erschien beiden sogar möglich, das DPE via Internet im begrenzten Rahmen fortzuführen.

Im Rahmen der Studie wurde auch der Fragestellung nachgegangen, ob die CBASP@home-Intervention zu einer Ver- 
besserung der Fähigkeit führt, die SA kriteriengerecht durchzuführen und wie sich diese Leistung zur depressiven Symptomatik verhält. Auffällig sind Differenzen im Leistungsniveau beim Übergang von stationärer Therapie zu CBASP@home. So entsprach die Leistung der SA bei beiden Patienten zu Beginn von CBASP@home nicht der am Ende der stationären Therapie erzielten Leistung. Der Leistungsabfall könnte sich dadurch erklären lassen, dass die Patienten die SA zu Hause völlig eigenständig und ohne therapeutische Hilfestellung erstellten. Nicht auszuschließen ist auch das vermehrte Auftreten dysfunktionaler Gedanken bedingt durch den Wegfall des geschützten stationären Rahmens, die Konfrontation mit den Alltagbelastungen sowie eine Verschlechterung der depressiven Symptomatik. Diese Veränderungen könnten sich in den «Interpretationen» sowie dem Handeln der Patienten manifestieren und sich so auf die Leistung in der SA auswirken. Es wäre interessant zu prüfen, ob im Rahmen von CBASP@ home aktuellere und stärker belastende Situationen für die SA herangezogen wurden als während der stationären Therapie. Möglicherweise fiel es den Patienten deswegen schwerer, die Situationen kriteriengerecht zu analysieren. Die beobachteten Differenzen in der Patientenleistung sind nicht abschließend zu erklären. Jedoch unterstreicht der Leistungsabfall die eingangs aufgestellte Vermutung, dass Patienten gegebenenfalls noch mehr Unterstützung bei dem Transferprozess der erlernten CBASP-Techniken in den Alltag benötigen könnten. Auch Strunk (2010, persönliche Mitteilung) ermittelte im Rahmen einer Evaluation der CBASP-Gruppe «DO!», in der ebenfalls regelmäßig SA durchgeführt wurden, dass die Patienten die Frage «Ich kann in der Gruppe Besprochenes oder Erarbeitetes im Alltag umsetzen» durchschnittlich nur mit «befriedigend» bewerteten.

In den hier vorgestellten Fallberichten stieg die Leistung in der SA auf das höchstmögliche Niveau an, wobei eine zeitgleiche Reduktion der depressiven Symptomatik beobachtet werden konnte (Abb. 2). Trotz des Leistungsabfalls während des Übergangs zwischen stationärem Aufenthalt und Nachsorge konnten beide Patienten ihre Fähigkeit in der kriteriengerechten Durchführung der SA während der Nachsorge stabilisieren. Positiv erscheint auch, dass Frau Rosenzweig zum Ende der stationären Behandlung keine Symptomverbesserungen aufwies, am Ende der Nachsorgeintervention jedoch remittierte. Diese Befunde lassen auf die Effektivität von CBASP@home bezüglich der Stabilisierung von chronisch depressiven Patienten hoffen. Aufgrund des gewählten Studiendesigns lassen sich Symptomverbesserungen jedoch nicht allein aufCBASP@home zurückführen.

Computerbasierte Interventionen scheinen in der Behandlung depressiver Patienten prinzipiell effektiv zu sein [z.B. Anderson und Cuijpers, 2009; Andrews et al., 2010; Cuijpers et al., 2013; Holländare et al., 2011; Richards und Richardson, 2012]. Die Studie von Wiersma und Kollegen [2011] enthält einen Hinweis darauf, dass chronisch depressive Patienten hinsichtlich der Symptomreduktion in ähnlichem Maße profi- tieren können wie episodisch Depressive, jedoch scheinen chronisch Depressive seltener zu remittieren (22,8 vs. 53,3\%). Dies hebt die vergleichsweise schwierige Behandlung der im Rahmen dieser Studie angesprochenen Patientengruppe hervor. Auch in der Nachsorge wurden Onlineinterventionen erfolgreich implementiert [z.B. Bauer et al., 2011, Ebert et al., 2013a,b; Zimmer et al., 2010]. Da die in dieser Studie vorgestellten sowie andere Forschungsergebnisse eine mögliche Wirksamkeit der Onlineintervention für chronisch depressive Patienten andeuten, kann vermutet werden, dass diese Strategie in der Tat eine effektive Therapieoption in der Stepdown-Behandlung chronisch depressiver Patienten darstellt.

Die Zunahme an Onlineinterventionen in der Nachsorge scheint den Bedarf an einfach zugänglichen und vermutlich auch kostengünstigen Therapieformen widerzuspiegeln. Das Pilotprojekt CBASP@home stößt seit seiner Implementierung bei Patienten auf reges Interesse. Viele stationäre Patienten möchten sehr gerne nach der Entlassung an dieser Nachsorge teilnehmen. Jedoch musste wegen personeller Engpässe nach 4 Pilotpatienten die Intervention zunächst pausieren. Die technische Umsetzung scheint stabil zu funktionieren und bietet auch Patienten mit nur geringen Computer- und/oder Internetkenntnissen die Möglichkeit, an der Intervention teilzunehmen. Bisher wird keine Onlineintervention routinemäßig angeboten, die sich über ein Symptom-Monitoring hinaus an die Patientengruppe der chronischen Depressiven in der Nachsorge richtet. Daher könnte CABSP@home zukünftig eine bedeutende Versorgungslücke schließen.

Die vorliegenden Einzelfallanalysen geben Anlass zur Hoffnung, dass CBASP@home als effektive Strategie der Step-down-Behandlung chronisch depressiver Patienten durchführbar ist und von ihnen angenommen wird. In kommenden Studien sollte weiterführend untersucht werden, ob sich diese Ergebnisse auch bei größeren Stichproben in einem randomisierten kontrollierten Studiendesign zeigen. Zudem sollte künftig sorgsam abgewogen werden, für welche Patienten CBASP@home unter welchen Bedingungen geeignet sein könnte. Interessant wäre insbesondere zu untersuchen, wie CBASP@home bei Patienten mit unterschiedlichem Ansprechen auf die Hauptbehandlung wirkt und ob Unterschiede je nach paralleler psychotherapeutischer Anbindung bestehen. Ein regelmäßiges Symptom-Monitoring sowie ein gut ausgearbeiteter Kriseninterventionsplan ist vonnöten, um auch $\mathrm{Pa}$ tienten mit ungünstigen Symptomverläufen und Suizidalität eine angemessene Hilfe bieten zu können.

Bei Berücksichtigung der gesamten Studienlage [vor allem Cuijpers et al., 2013; Ebert et al., 2013a,b; Holländare et al., 2011; Lin et al., 2013] kann vermutet werden, dass internetbasierte Nachsorgeinterventionen wie CBASP@home eine machbare, von Patienten akzeptierte und wirksame Vorgehensweise sind, um die Erfolge stationärer Behandlungen langfristig zu stabilisieren. Die Vorteile dieser Step-downProgramme scheinen in der Möglichkeit der systematischen Einführung oder Schulung hinsichtlich des Nachsorgepro- 
gramms bei weiterer Nutzung der während des stationären Rahmens aufgebauten therapeutischen Beziehung zu liegen. Zukünftig sollten diese Interventionen daher weiter beforscht und disseminiert werden.

\section{Disclosure Statement}

Die Autoren erklären hiermit, dass keine Interessenskonflikte in Bezug auf das Manuskript bestehen.

\section{Literatur}

Andersson G, Cuijpers P: Internet-based and other computerized psychological treatments for adult depression: a meta-analysis. Cogn Behav Ther 2009;38:196-205.

-Andrews G, Cuijpers P, Craske MG, McEvoy P, Titov $\mathrm{N}$ : Computer therapy for the anxiety and depressive disordes is effective, acceptable and practical health care: a meta-analysis. PLoS One 2010;5:1-6.

Arnow BA, Constantino MJ: Effectiveness of psychotherapy and combination treatment for chronic depression. J Clin Psychol 2003;59:893-905.

Bauer S, Wolf M, Mößner M, Zimmer B, Kordy H: Das Potenzial neuer Medien für Prävention und Psychotherapie. Stepped care in der psychosozialen Versorgung. Verhaltenstherapie und Verhaltensmedizin 2008;29:227-242.

Bauer S, Wolf M, Haug S, Kordy H: The effectiveness of internet chat groups in relapse prevention after inpatient psychotherapy. Psychother Res 2011;21: 219-226.

Beck AT, Steer RA, Brown GK: Beck Depression Inventory-II. Manual. San Antonio, The Psychological Corporation, 1996.

Brakemeier EL, Hautzinger M: Kognitive Verhaltenstherapie (KVT); in Bschor T (ed): Behandlungsmanual therapieresistente Depression. Stuttgart, Kohlhammer, 2008, pp 316-329.

-Brakemeier EL, Engel V, Schramm E, Schmidt T, Zobel I, Hautzinger M, Berger M, Normann C: Feasibility and outcome of cognitive behavioral analysis system of psychotherapy (CBASP) for chronically depressed inpatients: a pilot study. Psychother Psychosom 2011;80:191-194.

Brakemeier E-L, Normann C: CBASP Praxisbuch. Weinheim, Beltz, 2012.

Brakemeier E-L, Hautzinger M, Schramm E: Chronische Depression. Göttingen, Hogrefe, 2012a.

Brakemeier EL, Breger V, Spitzer C: Nebenwirkungen von ambulanter und stationärer Psychotherapie. Verhaltenstherapie und psychosoziale Praxis 2012b;41:569-578.

Bschor T: Behandlungsmanual therapieresistente Depression. Stuttgart, Kohlhammer, 2008.

Cukrowicz K, Joiner T: Computer based prevention of anxious and depressive symptoms in a non-clinical population. Cogn Ther Res 2007;31:677-693.

Cuijpers P, van Straten A, Schuurmans J, van Oppen P, Hollon SD, Andersson G: Psychotherapy for chronic major depression and dysthymia: a metaanalysis. Clin Psychol Rev 2010;30:51-62.

Cuijpers P, Lutz W, Riper H, Berger T, Berking M, Botella Arbona C, Banos Rivera RM, Araya R, Barkham M, Calbring P, Anderson G: Position statement: e-health and innovation of mental health care. Eur Neuropsychopharm 2013; (in press).

de Jong Meyer R, Hautzinger M, Kühner C, Schramm E: Evidenzbasierte Leitlinie zur Psychotherapie affektiver Störungen. Göttingen, Hogrefe, 2007.

Dunner DL: Acute and maintenance treatment of chronic depression. J Clin Psychiatry 2001;62 (suppl 6):10-16.
Ebert D, Hannig D, Tarnowski D, Sieland B, Meier H, Berking M: Web-basierte Rehabilitationsnachsorge nach stationärer psychosomatischer Therapie (W-RENA). Die Rehabilitation 2013a;52:164-172.

Ebert D, Tarnowski T, Gollwitzer M, Sieland B, Berking $\mathrm{M}$ : A transdiagnostic internet-based maintenance treatment enhances the stability of outcome after inpatient cognitive behavioral therapy: a randomized controlled trial. Psychother Psychosom 2013b;82:246-256.

Haaga DA: Introduction to the special section on stepped care models in psychotherapy 2000;68:547-548.

Hamilton M: A rating scale for depression. J Neurol Neurosur Ps 1960;23:56-61.

Hamilton M: Development of a rating scale for primary depressive illness. Br J Soc Clin Psychol 1967;6: 278-296.

Hautzinger M, Bailer M, Worall H, Keller F: BeckDepressions-Inventar (BDI). Bearbeitung der deutschen Ausgabe. Testhandbuch. Bern, Huber, 1994

Helbig-Lang S, Petermann F: Zum empirischen Aussagegehalt des Konzeptes «Chronische Depression». Z Psychiatr Psychol Psychother 2009;57:235-243.

Holländare F, Johnsson S, Randestad M, Tillfors M, Carlbring P, Andersson G, Engström I: Randomized trial of internet-based relapse prevention for partially remitted depression. Acta Psychiatr Scand 2011;124:285-94.

Kanfer FH, Saslow G: Verhaltenstheoretische Diagnostik; in Schulte D (ed): Diagnostik in der Verhaltenstherapie. München, Urban und Schwarzenberg, 1976, pp 24-59.

Keller MB, McCullough JP, Klein DN, et al.: A comparison of nefazodone, the cognitive behavioralanalysis system of psychotherapy, and their combination for the treatment of chronic depression. $\mathrm{N}$ Engl J Med 2000;342:1462-1470.

Klein DN, Santiago NJ, Vivian D, et al.: Cognitive-behavioral analysis system of psychotherapy as a maintenance treatment for chronic depression. J Consult Clin Psychol 2004:72:681-688.

Kordy H: Internet- und mediengestützte Therapie. Psychother Psych Med 2013;63:12-18.

Kordy H, Golkaramnay V, Wolf M, Haug S, Bauer S: Internetchatgruppen in Psychotherapie und Psychosomatik: Akzeptanz und Wirksamkeit einer Internet-Brücke zwischen Fachklinik und Alltag. Psychotherapeut 2006;51:144-153.

Ley P, Helbig-Lang S, Petermann F: Kann man chronische Depression effektiv psychotherapeutisch behandeln? Z Psychiatr Psychol Psychother 2009; 57:253-263.

Lin J, Ebert D, Berking M, Lehr D, Baumeister H: Internetbasierte kognitiv-behaviorale Behandlungsansätze: State of the Art und Einsatzmöglichkeiten in der Rehabilitation. Die Rehabilitation 2013;52: 155-163.

Manber R, Arnow B, Blasey C, et al.: Patient's therapeutic skill acquisition and response to psychotherapy, alone or in combination with medication. Psychol Med 2003:33:693-702.
McCullough JP: Treatment for Chronic Depression: Cognitive Behavioral Analysis System of Psychotherapy (CBASP). New York, Guilford, 2000.

McCullough JP Jr., Lord BD, Conley KA, Martin AM: A method for conducting intensive psychological studies with early onset chronically depressed patients. Am J Psychother 2010;64:317-338.

Piaget J: Intelligence and Affectivity: Their Relationship During Child Development. Palo Alto, Annual Reviews, 1981.

Richards D, Richardson T: Computer-based psychological treatments for depression: a systematic review and meta-analysis. Clin Psychol Rev 2012;32: 329-342.

Riso LP, Miyatake RK, Thase ME: The search for determinants of chronic depression: a review of six factors. J Affect Disord 2002;70:103-115.

Santiago NJ, Klein DN, Vivian D, et al.: The therapeutic alliance and CBASP-specific skill acquisition in the treatment of chronic depression. Cogn Ther Res 2005;29:803-817.

Schramm E, Caspar F, Berger M: Spezifische Therapie für chronische Depression. Nervenarzt 2006;77: 355-371.

Schweiger U, Sipos V, Hohagen F: Diagnostik und Therapie chronischer Depressionen. Psychiatr Psychother up2date 2007;1:117-129.

Schweiger U, Sipos V: Situationsanalyse nach dem Modell des Cognitive Behavioral Analysis System of Psychotherapy (CBASP). Verhaltenstherapie und Verhaltensmedizin 2009;30:56-68.

Wiersma JE, Titov N, van Schaik DJ, van Oppen P, Beekman AT, Cuijpers P: Treating chronic symptoms of depression in the virtual clinic: findings on chronicity of depression in patients treated with in ternet-based computerized cognitive behaviour therapy for depression. Psychother Psychosom 2011;80:313-315.

Wolf M, Maurer W-J, Dogs P, Kordy H: E-Mail in der Psychotherapie - ein Nachbehandlungsmodell via Electronic Mail für die stationäre Psychotherapie. Psychother Psych Med 2006;56:138-146.

Wolf M, Zimmer B, Dogs P: Chat- und E-Mail-Brücke: Nachsorge nach stationärer Psychotherapie; in Bauer S (ed): E-Mental Health. Neue Medien in der psychosozialen Versorgung. Heidelberg, Springer, 2008, pp 219-235.

Zimmer B, Moessner M, Kordy H: Die Kommunikation von Patienten mit einer chronischen Erkrankung in einem Internet-Chat zur Nachsorge einer stationären psychosomatischen Behandlung. Die Rehabilitation 2010;49:301-307.

Zimmerman M, Chelminski I, Posternak M: A review of studies of the Hamilton Depression Rating Scale in healthy controls. J Nerv Ment Dis 2004;192:595601. 